\title{
Speed-accuracy tradeoff in double stimulation: Effects on the first response*
}

\author{
JAMES L. KNIGHT, 'JR. and BARRY H. KANTOWITZ† \\ Purdue University, West Lafayette, Indiana 47907
}

\begin{abstract}
A single-stimulation and two double-stimulation response conditions were compared using explicit payoff matrices to vary speed-accuracy tradeoff. Under accuracy payoff, response latency (RT, ) to the first stimulus increased as ISI dropped but accuracy remained high and relatively constant. Under speed payoff, $\mathbf{R T}_{1}$ was only slightly affected by ISI but accuracy dropped as ISI decreased. Transmitted information rates consistently reflected detrimental effects of short ISI. In double stimulation, but not in single stimulation, error response latency exceeded correct response latency. Furthermore, error response latencies were found to be far more variable and more sensitive to changes in speed-accuracy condition than were correct response latencies. Finally, under both speed and accuracy conditions, response latency to the first of two successive stimuli was faster if a response was also required to the second stimulus. Implications of the data for possible models of double-stimulation speed-accuracy tradeoff are considered.
\end{abstract}

As Ss become more practiced at a task, both speed and accuracy of performance increase (Fitts, 1966). However, at a particular level of practice, Ss exhibit the ability to sacrifice response speed for increased accuracy or vice versa (Fitts, 1966; Hale, 1969; Pachella \& Pew, 1968). This speed-accuracy tradeoff (SAT) has been a center of both empirical and theoretical interest (Edwards, 1961, 1965; Fitts, 1966). However, research has concentrated primarily upon the effects of SAT in single-stimulation choice reaction time (CRT) paradigms. In such studies, Ss are typically presented with a single stimulus from a set of possible stimuli and must select and execute an appropriate response. The $\mathrm{E}$ attempts to manipulate SAT by emphasizing the relative importance of the two performance dimensions, speed and accuracy.

A multitude of models has developed to deal with the extensive body of SAT data in single-stimulation CRT (Edwards, 1965; Fitts, 1966; Ollman, 1966; Ollman \& Billington, 1972; Stone, 1960; Yellott, 1971). These models may be divided into two general groups. The first, stimulus sampling models (Edwards, 1965; Stone, 1960), assume that Ss accumulate information about a presented stimulus over time. The longer Ss delay their response, the more information is available to them. Consequently, response accuracy increases continuously with latency.

The second general class of single-stimulation SAT models, fast guess models (Ollman, 1966; Yellott, 1971), views the SAT as a mixture of two response modes. In one mode, only minimal information processing is performed on the stimulus. Instead, Ss execute a guess response upon detection of stimulus onset or at a preset

*This research was supported in part by NIMH Grant MH 21169. This report is based on a thesis submitted by the first author in partial fulfilment of the requirements for the MS degree and supervised by the second author.

tRequests for reprints may be addressed to either author, Department of Psychological Sciences, Purdue University, West Lafayette, Indiana 47907. temporal deadline. This response mode, though very fast, is often inaccurate. In the other response mode, more complete stimulus processing is done and Ss execute a slower but accurate "stimulus-controlled response" (Yellott, 1971). By varying the mixture of these two response modes, Ss can generate an SAT over a series of trials.

Despite these developments in single-stimulation CRT, the role of SAT in double-stimulation CRT remains largely unexplored. In double stimulation, Ss are presented with two stimuli (S1 and S2) separated by a short temporal interstimulus interval (ISI). Responses may be required to $S 1$ (i.e., R1), to $S 2$ (i.e., R2), or to both $\mathrm{S} 1$ and $\mathrm{S} 2$. In double stimulation, a psychological refractory period (PRP) effect is often found in which reaction time to $S 2$ (i.e., $R_{2}$ ) increases as ISI decreases. Evidence also suggests that $\mathrm{RT}_{1}$ may be similarly affected (Herman \& Kantowitz, 1970). In addition, while single-stimulation CRT data generally show error responses to be faster than correct responses (e.g., Hale, 1969; Howell \& Kreidler, 1963), double-stimulation CRT results often show error response to be slower (Kantowitz, 1969, 1972). The general aim of the present study was to examine the influence of SAT on double-stimulation effects, particularly those involving the response to the first stimulus.

From an empirical standpoint, SAT is important in both single- and double-stimulation because it may interact with intended experimental variables and distort their effects. Ss' freedom to vary their position on the speed-accuracy characteristic represents an unwanted source of variation both between and within Ss. Edwards (1961) has discussed this problem in relation to single stimulation and pointed out that comparison between experimental conditions may be misleading if Ss were not performing under comparable speed-accuracy demands. Thus, a faster RT in one condition may reflect only Ss' willingness to perform less accurately. While 
recognition of this fact has been apparent in single-stimulation literature, reports of double-stimulation studies have concentrated upon one dependent variable-RT-to the neglect of accuracy. Double-stimulation accuracy data is seldom presented as a function of major experimental variables (e.g., Kantowitz, 1974; Helson \& Steger, 1962; Herman, 1969). Thus, the possible influence of SAT cannot be evaluated.

Some double-stimulation studies may exemplify the undesirable consequences of between-Ss variation in SAT. Helson and Steger (1962) investigated the effect of an S2 not requiring a response upon a two-choice R1. While finding $\mathrm{RT}_{1}$ increases attributable to $\mathrm{S} 2$, they report that their Ss could be divided into two distinct groups: those affected by $\mathrm{S} 2$ and those not affected by S2. One might conjecture that these groups represent performance at differing SATs. Were this true, one would expect accuracy differences as well between these groups. However, these data were not presented. Nickerson (1967) also presented double-stimulation data which contain a similar dichotomy. For two Ss in Experiment VI (A and B, Fig. 2, p. 309) $\mathrm{RT}_{1}$ was almost unaffected by ISI, while Ss C, D, E, and F exhibit $\mathrm{RT}_{1}$ curves which decrease with ISI in a fashion reminiscent of $\mathrm{RT}_{2}$ PRP functions. Finally, Herman (1969) failed to find expected $\mathrm{RT}_{1}$ changes in an experimental group, $\mathrm{C}_{\max }$, as a function of ISI. However, performance changes may have been apparent in error rates which were not reported.

Similarly, double-stimulation results reported by Herman and McCauley (1969) may have been influenced by differences in speed-accuracy requirements. Examining the effects of the second response (R2) upon the first (R1), they found $\mathrm{RT}_{1}$ to be faster for a group required to make both $\mathrm{R} 1$ and $\mathrm{R} 2$ than for a group required to execute only $R 1$. However, their methodology provided a 50-msec "grace period" for the R1-R2 group, so that some responses made by these Ss and scored as correct would have been considered errors in the single-response group. Thus, $\mathrm{Ss}$ in the R1-R2 group may have achieved a faster $\mathrm{RT}_{1}$ because of the more lenient accuracy requirement implicitly imposed upon them.

From a theoretical standpoint, little attempt has been made to incorporate SAT mechanisms into existing models of double-stimulation performance. Preliminary steps have been taken by Annett (1966), who developed a model which attributes the PRP to SAT effects rather than to sequential processing limitations. According to this model, Ss attempt to maximize their "payoff" by adjusting response speed. Annett claimed that an implicit payoff structure is present in the typical double-stimulation paradigm with random ISI presentation. For anticipatory $\mathrm{R} 2 \mathrm{~s}$ a penalty is assessed which increases with the R2-S2 interval. However, a reward which decreases with the S2-R2 interval is given for legal R2s. With random ISI presentation, S's expected payoff would be maximized if R2 were consistently timed to coincide with the longest ISI. The first stimulus would provide a temporal marker to initiate timing. While this model suggests the possible importance of SAT in double stimulation, it seems incomplete since PRP effects are found even if ISI is presented in constant blocks. Furthermore, changes in $\mathrm{RT}_{1}$ as a function of ISI are unexplained.

It may be possible to provide existing double-stimulation models with an SAT mechanism by application of a single-stimulation SAT model to each component $\mathrm{S}-\mathrm{R}$ decision in the double-stimulation paradigm. Using a stimulus sampling model, one might expect the range of SAT to be a function of ISI and bounded by the maximum ISI. In many double-stimulation paradigms, the interval during which Ss can accumulate relevant information is inherently limited by the task itself, as when correct responses are determined by the temporal ordering of stimuli. For instance, $S$ may be required to press a key corresponding to the first or second of two sequentially presented lights. Once both lights have been illuminated, additional information concerning temporal order can no longer be extracted from the stimulus display. Thus, SAT should be limited by ISI if a stimulus sampling mechanism is operating in double stimulation.

Generally, fast guess models (Ollman, 1966; Yellott, 1971) make strong predictions in both single- and double-stimulation contexts. First, error responses are expected to be faster than correct responses in both single and double stimulation. Second, error responses should exhibit RT density functions characterized by smaller variance than correct responses because correct responses represent a mixture of both fast lucky guesses and slow stimulus-controlled responses. Error responses, on the other hand, are more homogeneous. Finally, error RT density functions should not shift with SAT. Shift in SAT is accomplished by varying the proportion of guesses $S$ is willing to make, not by changing the RT characteristics of the guesses. A more recent variant of the fast guess model, the deadline model (Ollman \& Billington, 1972), makes the prediction that changes in error RT as SAT varies will be less than changes in correct RT. This follows from the assumption that $\mathrm{S}$ tries to set a deadline that is bounded below by the longest FP and above by the longest FP plus correct decision latency.

A final approach to double-stimulation SAT mechanisms lies in extension of current double-stimulation performance models. Herman and Kantowitz (1970) suggested that increases in $\mathrm{RT}_{1}$ at short ISIs eliminate all but one contending theory of double-stimulation performance-response conflict theory (Reynolds, 1966). This theory attributes latency increases at short ISIs to difficulty in selecting a response from a set of competing response tendencies. Response delay increases with the conflict thus generated. However, the detrimental effects of high 
conflict might appear as either increased latency or increased error rate, depending upon S's choice of position on his speed-accuracy operating characteristic. Since high conflict would be likely to cause Ss to vary response speed and accuracy over a wide range to minimize detrimental effects of such conflict, response conflict theory makes a prediction opposite to stimulus sampling models: The range of SAT should be greatest at short ISIs, since conflict is highest when ISI is smallest ${ }^{1}$ (Herman \& Kantowitz, 1970; Kantowitz, 1969).

The present experiment was designed to examine two common double-stimulation response conditions and one single-stimulation response condition under two levels of speed-accuracy requirements. The response conditions were chosen to focus on $\mathrm{RT}_{1}$ effects caused by changes in SAT. Because the response conditions of the present experiment replicate those employed by Herman and McCauley (1969), it will also be possible to assess the role of SAT in their results.

\section{METHOD}

\section{Subjects}

Sixty female undergraduates enrolled in an introductory psychology course served as Ss to fulfill a course requirement. Ss were randomly assigned to one of six experimental groups.

\section{Apparatus}

The experiment was controlled via a LINC-8 computer. Stimuli were presented on a remote Tektronix Type 602 display scope. The scope, equipped with a high-speed P-20 phosphor, was located in a testing room adjoining the computer room. Responses were made by pressing specially designed piano-type keys. Key closure required a movement distance of $1 / 16$ in. $(1.58 \mathrm{~mm})$ and a static force of $60 \mathrm{~g}$. Responses were monitored and recorded automatically by the computer. A crystal-controlled General Radio Model 1217-C pulse generator provided an accurate time base for measuring RT to the nearest millisecond. The LINC-8 recorded data on punched paper tape.

\section{Design}

Six experimental groups of $10 \mathrm{Ss}$ each were employed. These groups represented all combinations of two speed-accuracy conditions and three response conditions. Speed-accuracy conditions (SPEED or ACCUR) were determined by two explicit payoff matrices which differentially rewarded either response speed or response accuracy as shown in Fig. 1. The point values chosen for the payoff matrices were selected on the basis of pilot studies with a separate group of Ss. The fairly extreme values shown in Fig. 1 reflect the difficulty encountered in inducing any change in error rates with the unpracticed Ss employed in both the pilot and the main study.

Response conditions chosen were single stimulation (SS), double stimulation, respond only to S1 (DS-1), and double stimulation, respond to both S1 and S2 (DS-2). The six experimental groups were thus coded: SS[SPEED], SS[ACCUR], DS-1[SPEED], DS-1[ACCUR], DS-2[SPEED], DS-2[ACCUR].

Foreperiod (FP) duration (onset of warning signal to S1 onset) could assume any of four equiprobable values: $1.5,1.75$, $2.25,2.5 \mathrm{sec}$. S1 was an $\mathrm{X}$ presented on the display scope to either the left or right of a central fixation line. S1 occurred equiprobably on either side of the line. Thus, 1 bit of S1-R1 uncertainty was obtained. Each trial was characterized by one of eight combinations of S1 (LEFT or RIGHT) and FP. Ss had no foreknowledge of the combination to be presented on any trial.

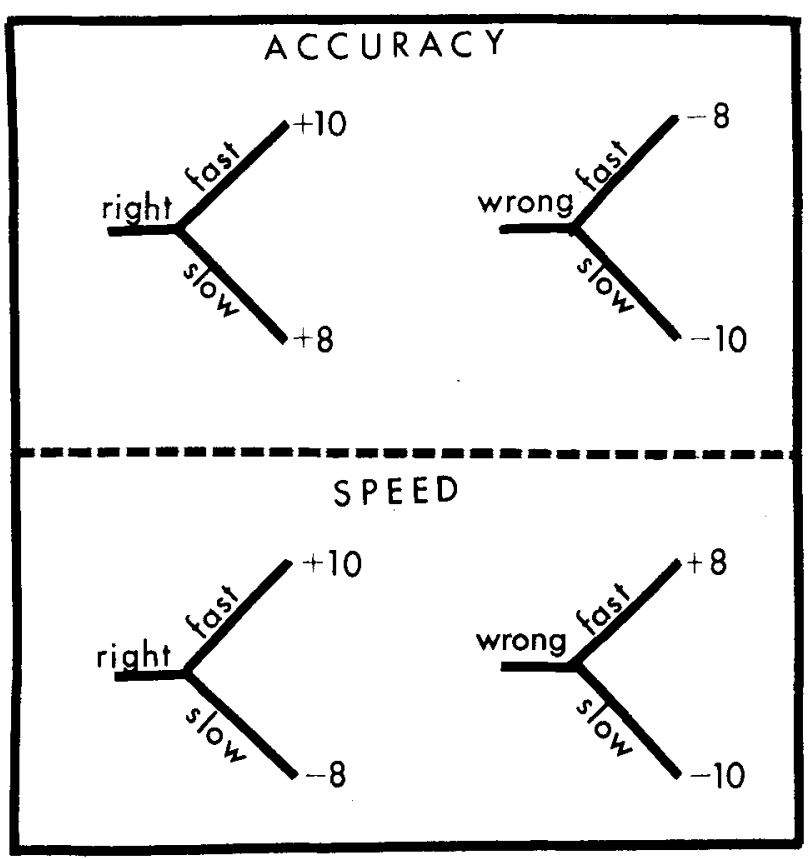

Fig. 1. Payoff matrices presented to Ss.

These eight combinations were arranged in five random sequences of 32 trials. Each S1-FP combination occurred four times. In addition, each sequence was preceded by four practice trials, during which each S1 appeared twice and each FP was used once,

These five single-stimulation sequences were also transformed into a yoked set of five double-stimulation sequences by adding an S2 on all trials; S2 consisted of an X on the opposite side of the fixation line from $S 1 . S 1$ and $S 2$ were easily discriminable. One of five ISI values $(33,66,132,264$, or $528 \mathrm{msec})$ was assigned to each of the five double-stimulation sequences. Thus, each 36-trial double-stimulation sequence was characterized by constant ISI.

The five yoked single- and double-stimulation sequences were arranged according to a 5 by 5 Latin square into five presentation orders. Thus, for double-stimulation sequences this produced five orders of ISI presentation. Each S experienced one order of five trial sequences during the experiment. Within experimental groups, two Ss were randomly assigned to each of the five sequence orders.

\section{Procedure}

Ss were tested individually in a dim testing room. Ss sat approximately $3.3 \mathrm{ft}(1 \mathrm{~m})$ in front of the eye-level display scope used for stimulus presentation. After familiarizing $\mathrm{S}$ with the equipment, $E$ left the testing room and read instructions to $S$ over a two-way Fannon intercom. The instructions indicated that a warning signal consisting of a single vertical line $(2.54 \mathrm{~cm}$ high) would be presented on the scope. Depending upon response condition, $S$ was told that either one $X(2.54 \mathrm{~cm}$ high and wide) would appear on one side of the warning line or that two Xs would appear in rapid succession on alternate sides of the line. Then $S$ was told to press the response key corresponding to the side of the warning line on which the single $X$ appeared (SS), to press the key corresponding to the side of the first $X$ and ignore the second $X$ (DS-1), or to press the key corresponding to the side of the first $\mathrm{X}$ and then press the key corresponding to the side of the second $X$ when it appeared (DS-2).

When S made correct responses, the corresponding Xs were removed from the display screen. Two and one-half seconds after S2 onset (2.632 sec after S1 onset for SS), the warning line and 
Table 1

Mean RT, (Milliseconds) for All Combinations of Response Condition and Speed-Accuracy Condition

\begin{tabular}{|c|c|c|c|c|}
\hline \multirow{2}{*}{$\begin{array}{c}\text { Speed- } \\
\text { Accuracy } \\
\text { Condition }\end{array}$} & \multicolumn{3}{|c|}{ Response Condition } & \multirow[b]{2}{*}{ Mean } \\
\hline & SS & DS-1 & DS-2 & \\
\hline Accuracy & 357 & 499 & 459 & 439 \\
\hline Speed & 317 & 400 & 340 & 352 \\
\hline Mean & 337 & 450 & 399 & 395 \\
\hline
\end{tabular}

Note-Means based on combined correct and error responses.

any remaining Xs were turned off. Feedback information was then displayed on the screen for 4 sec. Feedback included the score earned for each response of the trial as well as the cumulative score earned in the experiment. A 3-sec delay period was inserted between feedback offset and presentation of the warning line for the next trial. Ss were told that their performance in the task would be scored on a response by response basis and the $S$ who accumulated the highest total score in each experimental group would receive a $\$ 5$ bonus.

The payoff matrix used to score responses was presented to $S$ as a tree diagram (Fig. 1) on a $5 \times 8$ in. white index card which lay beside the response keys. Ss were told that anticipatory responses (responding prior to stimulus onset) would be heavily penalized ( -12 points) whether or not correct. Also, in response conditions requiring only one response, if $S$ responded twice a similar penalty was assessed. In Response Condition DS- 2 both $\mathrm{R} 1$ and $\mathrm{R} 2$ were scored individually according to the same payoff matrix. The accumulated score for these Ss was, therefore, generally twice that of Ss in the single-response conditions. Also, in Response Condition DS-2 responses had to occur in correct temporal order to be correct, since no grace period (Herman \& McCauley, 1969) was used.

A criterion time was used to classify RTs as fast or slow. This criterion time was initially set to $300 \mathrm{msec}$. Once the experiment began, the computer interactively shifted the criterion time in 20 -msec steps to maintain a ratio of fast to slow responses of .80. This was done to account for individual differences and differences in mean RT at different ISIs. A separate criterion was maintained and independently adjusted for $\mathrm{RT}_{2}$. Ss were given a short rest period between each block of 36 trials. The entire experiment required about $45 \mathrm{~min}$ per $\mathrm{S}$.

\section{RESULTS}

The first four trials of each 36-trial block were intended as practice and not analyzed. All poststimulus responses were scored. On single-stimulation trials, if $S$ responded twice, the first response was always scored as an error. Such trials were infrequent in all single-response groups $(<.5 \%)$.

\section{Effects on the First Response}

R1 Latency. The effects of speed-accuracy condition and response condition on $\mathrm{RT}_{1}$ may be seen in Table 1 . Figure 2 depicts the effects of these variables as a function of ISI. Table 1 and Fig. 2 are based on both correct and error responses. ${ }^{2}$ The pooled correct-error $\mathrm{RT}_{1}$ data were submitted to a five-way mixed analysis of variance (between variables: speed-accuracy condition, response condition; within variables: ISI, FP, repetitions). Both speed-accuracy condition and response condition produced significant effects $[F(1,54)$ $=12.81, p<.001$ and $F(2,54)=7.29, p<.005$, respectively]. Under accuracy payoff, mean RT was $84 \mathrm{msec}$ slower than under speed payoff. The difference between speed and accuracy conditions was greatest for Response Condition DS-2, $119 \mathrm{msec}$, and smallest for the single-stimulation control, SS, $40 \mathrm{msec}$. However, the Speed-Accuracy by Response Condition interaction failed to reach significance $[F(2,54)<1]$. For double-stimulation groups, speed-accuracy condition produced the greatest changes in $\mathrm{RT}_{1}$ at the shortest ISIs, 33 and $66 \mathrm{msec}$. For longer ISIs the change in $\mathrm{RT}_{1}$ was approximately constant. A separate analysis of variance conducted on data from only double-stimulation groups showed the Speed-Accuracy by ISI interaction (Fig. 2) to be significant $[F(4,144)=$ $6.86, p<.01]$. No other two-way interactions with speed-accuracy condition were statistically significant. The three-way interaction, Speed-Accuracy by Response

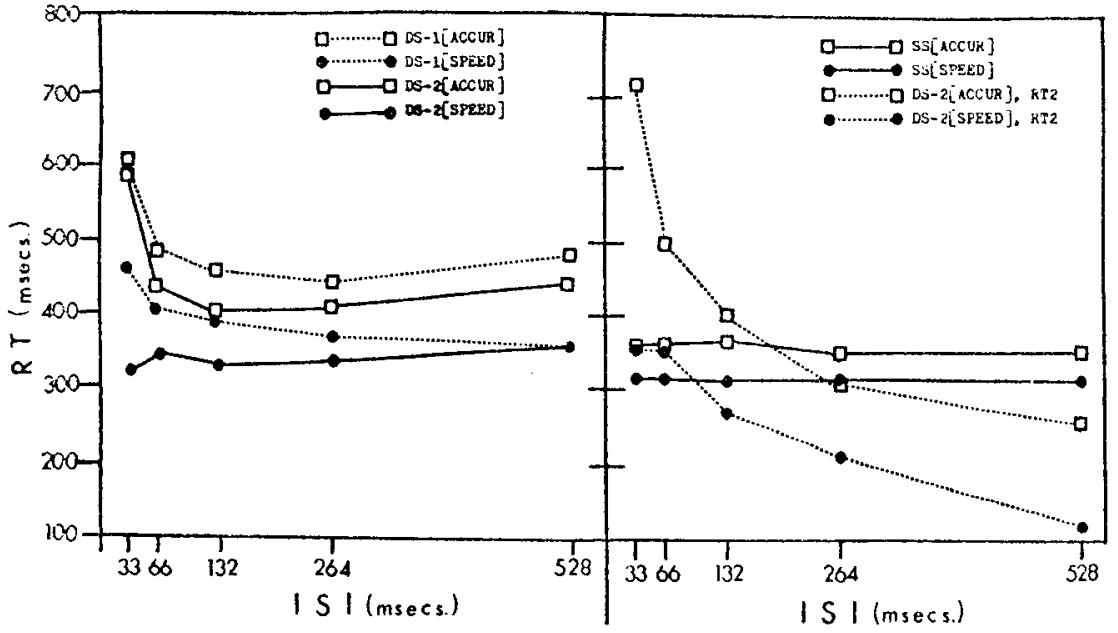

Fig. 2. Left panel: $\mathrm{RT}_{1}$ as a function of ISI for double-stimulation groups only. Right panel: RT $_{2}$ for DS-2 groups (dotted line) and $R T_{1}$ for single-stimulation groups (solid lines) as a function of yoked (pseudo-ISI) blocks. 
Condition by ISI, shown in Fig. 2 was reliable $[F(8,216)$ $=6.72, \mathrm{p}<.01] ;$ this interaction is primarily attributable to the lack of ISI effect in the single-stimulation response conditions.

Figure 2 shows that generally $\mathrm{RT}_{1}$ increased as ISI decreased $[F(4,216)=14.31, p<.001]$. The elevation in $\mathrm{RT}_{1}$ is similar to that often observed for $\mathrm{RT}_{2}$ in studies of the PRP. At the longest ISI $(528 \mathrm{msec}), \mathrm{RT}_{1}$ increased slightly for Groups DS-1 [ACCUR], DS-2[ACCUR], and DS-2 [SPEED]. A Newman-Keuls test indicated that, for each of these groups, $\mathrm{RT}_{1}$ at $528 \mathrm{msec}$ was not statistically different from the fastest $\mathrm{RT}_{1}$ on the respective curve, $\mathrm{q}_{\mathrm{r}} \mathrm{s}(2,216) \leqslant 2.34, \mathrm{p}>.05$. Nevertheless, similar increases have been reported elsewhere (e.g., Reynolds, 1966).

In contrast to the general increase in $\mathrm{RT}_{1}$ with decreasing ISI is the striking lack of ISI effect in the DS-2[SPEED] group. A simple effects test for ISI within Group DS-2[SPEED] failed to show statistical significance $[F(4,36)=1.71, p>.05]$. To determine if this function might reflect an over-representation of characteristically fast error responses, a corresponding function based on correct responses only was constructed. This was essentially identical to Fig. 2 . The greatest difference between the functions, $11 \mathrm{msec}$, occurred not at the short ISIs (where most errors occurred) but at the longest, 528 msec. Thus, the lack of ISI effect cannot be attributed to error responses alone. Figure 2 shows that this same response condition, DS-2, when performed under accuracy payoff was strongly affected by ISI. The finding that increases in $\mathrm{RT}_{1}$ due to ISI may be eliminated under speed payoff may account for the dichotomies reported by Helson and Steger (1962) and Nickerson (1967). The effect of ISI upon $\mathrm{RT}_{1}$ does depend upon the SAT adopted by $\mathrm{S}$.

Within both speed and accuracy conditions, the ordering of Response Conditions SS, DS-2, and DS-1 from fastest to slowest $\mathrm{RT}_{1}$ confirmed the results of Herman and McCauley (1969). Also, with one exception, double-stimulation groups differed significantly from their respective single-stimulation controls by Dunnette tests [ds $(54) \geqslant 1.99, \mathrm{p}<.05$ ] Group DS-2[SPEED] did not differ significantly from Group SS[SPEED] [d(54) $=.47, \mathrm{p}>.1]$. Though $\mathrm{RT}_{1}$ in DS-2 conditions was faster than RT ${ }_{1}$ in DS-1 conditions, a liberal $\mathrm{t}$ test failed to indicate statistical significance $\left[\mathrm{t}(54)=1.70, \mathrm{MS}_{\text {error }}=13951, .1>\mathrm{p}>\right.$ $.05]$. This contrasts with results of Herman and McCauley (1969). However, the present study used two ISIs (264 and $528 \mathrm{msec}$ ) that were considerably longer than any used by Herman and McCauley (1969). An additional analysis of variance restricted to ISIs of 33, 66 , and $132 \mathrm{msec}$ showed the difference between Response Conditions DS-1 (470 msec) and DS-2 $(406 \mathrm{msec})$ to be statistically significant $[\mathrm{t}(54)=2.22$, $\left.\mathrm{MS}_{\text {error }}=8107, \mathrm{p}<.05\right]$.

First response latency also varied as a function of FP such that RT $_{1}$ decreased $(404,396,393,389 \mathrm{msec})$ as
FP increased $(1.5,1.72,2.25,2.5 \mathrm{sec})[\mathrm{F}(3,162)=6.79$, $\mathrm{p}<.01]$. This FP effect was smallest at ISIs of 66 and $132 \mathrm{msec}$, giving rise to a significant FP by ISI interaction $[F(12,648)=1.89, p<.01]$. No other interactions with FP were observed. A significant effect of repetitions $[F(7,378)=3.75, p<.01]$ confirmed that $\mathrm{RT}_{1}$ became progressively faster within each 36-trial block.

Response latencies for incorrect first responses were examined within each experimental group. Comparison of correct and error response latencies is shown in Table 2. Single-stimulation response conditions produced few errors even under speed payoff and, consequently, conclusions based on single-stimulation error $\mathrm{RT}_{1}$ must be tentative. Also, Ss occasionally failed to respond within the allotted 2.5 -sec post-S2 interval (post-S1 interval in Response Condition SS). Such trials are excluded from Table 2. Thus, the total number of correct and error responses for each experimental group is generally less than the maximum possible (1600).

For single-stimulation response conditions, error $\mathrm{RT}_{1}$ was faster than correct $R_{1}$. This is consistent with fast guess type CRT models. This result also occurred in Group DS-2 [SPEED]. However, all other double-stimulation groups showed error $\mathrm{RT}_{1}$ to be slower than correct $\mathrm{RT}_{1}$. This result differs from data obtained in previous single-stimulation studies (e.g., Fitts, 1966; Hale, 1969; Yellott, 1971) but confirms double-stimulation results of Kantowitz $(1969,1972)$.

A deadline model (Ollman \& Billington, 1972) of R1 performance might hold that on long FP trials correct response latencies should exceed error response latencies, since the response deadline is often reached before S1 processing is complete. However, on short FP trials S1 processing will usually terminate before the deadline is reached. Consequently, error latency should, on the average, exceed correct response latency on short FP trials. The data of Table 2 were, therefore, partitioned by FP. This showed a pattern of results at all FPs highly similar to the data of Table 2 . Predictions of the deadline model were, therefore, not maintained in this case. Table 2 further shows that error $\mathrm{RT}_{1}$ in double stimulation was far more sensitive to changes in speed-accuracy condition than was correct $\mathrm{RT}_{1}$. However, in single-stimulation conditions error and correct response latencies decreased almost equally (50 msec) as performance emphasis shifted from accuracy to speed. The finding that error $\mathrm{RT}_{1}$ varied with speed-accuracy condition is in disagreement with fast guess models which assume that errors result only from preprogrammed guesses.

Probability density functions for correct and error $\mathrm{RT}_{1}$ were separately constructed for each combination of speed-accuracy condition and response condition. This set of 12 figures is presented in Knight (1972). The parameters of the 12 distributions are shown in Table 2. Examination of these figures (Knight, 1972) revealed that error functions for double-stimulation groups other 
Table 2

Parameters of Latency Distributions

\begin{tabular}{|c|c|c|c|c|c|}
\hline Group & Distribution - & $\mathbf{N}$ & SD & Mean & $\begin{array}{c}\text { Difference } \\
\text { (Error - Correct RT) }\end{array}$ \\
\hline SS[ACCUR] & $\begin{array}{l}\text { Corrects } \\
\text { Errors }\end{array}$ & $\begin{array}{r}1593 \\
7\end{array}$ & $\begin{array}{l}81.9 \\
58.4\end{array}$ & $\begin{array}{l}357 \\
327\end{array}$ & -30 \\
\hline SS[SPEED] & $\begin{array}{l}\text { Corrects } \\
\text { Errors }\end{array}$ & $\begin{array}{r}1496 \\
97\end{array}$ & $\begin{array}{l}68.0 \\
83.9\end{array}$ & $\begin{array}{l}310 \\
281\end{array}$ & $-29 *$ \\
\hline DS-1 [ACCUR] & $\begin{array}{l}\text { Corrects } \\
\text { Errors }\end{array}$ & $\begin{array}{r}1529 \\
68\end{array}$ & $\begin{array}{l}223.7 \\
367.8\end{array}$ & $\begin{array}{l}488 \\
702\end{array}$ & $214^{*}$ \\
\hline DS-1 [SPEED] & $\begin{array}{l}\text { Corrects } \\
\text { Errors }\end{array}$ & $\begin{array}{r}1366 \\
231\end{array}$ & $\begin{array}{l}125.9 \\
196.4\end{array}$ & $\begin{array}{l}386 \\
420\end{array}$ & $33^{*}$ \\
\hline DS-2 [ACCUR] & $\begin{array}{l}\text { Corrects } \\
\text { Errors }\end{array}$ & $\begin{array}{r}1545 \\
54\end{array}$ & $\begin{array}{l}168.9 \\
360.6\end{array}$ & $\begin{array}{l}449 \\
754\end{array}$ & $304^{*}$ \\
\hline DS-2 [SPEED] & $\begin{array}{l}\text { Corrects } \\
\text { Errors }\end{array}$ & $\begin{array}{r}1249 \\
350\end{array}$ & $\begin{array}{r}78.7 \\
128.6\end{array}$ & $\begin{array}{l}344 \\
325\end{array}$ & $-18^{*}$ \\
\hline
\end{tabular}

*Difference significant at $p<.01$ by unequal- $N$ t test. The following ERROR $R T$ comparisons were similarly significant: $D S-1[A C C U R]$ vS DS-1[SPEED] and DS-2[ACCUR] vs DS-2[SPEED].

than DS-2[SPEED] were quite uniform over a wide range. With the exception of Group DS-2 [SPEED], a clear mode was not found for any double-stimulation density function. This seems contrary to predictions of the fast guess class of CRT models. Group DS-2 [SPEED] did, however, produce a unimodal error density function with a peak at $260 \mathrm{msec}$. In contrast to most double-stimulation error density functions, single-stimulation groups produced unimodal functions. The mode changed as a function of speed-accuracy condition from $340 \mathrm{msec}$, SS [ACCUR], to $220 \mathrm{msec}$, SS [SPEED]. As shown in Table 2, error $\mathrm{RT}_{1}$ density functions for all experimental groups other than SS[ACCUR] were characterized by larger standard deviations than were correct $\mathrm{RT}_{1}$ functions. To some extent, this reflects the fact that fewer error than correct $\mathrm{RT}_{1}$ observations were used in computation of respective standard deviations. However, $95 \%$ confidence intervals were constructed for the standard deviations of correct and error $\mathrm{RT}_{1}$ functions within each experimental group. Overlap was found in only one group, SS [ACCUR].

R1 Accuracy. The effect of speed-accuracy condition and response condition upon R1 accuracy may be seen in Table 3; Fig. 3 displays the effects of these factors as a function of ISI. A five-way mixed analysis of variance similar to that employed with $\mathrm{RT}_{1}$ showed effects of speed-accuracy condition $[F(1,54)=46.24, p<.001]$, response condition $[F(2,54)=10.08, p<.01]$, and ISI $[F(4,216)=58.08, p<.001]$ to be significant. Changes in speed-accuracy condition produced the greatest change in percentage correct for Response Condition DS-2 $\left[q_{r}(5,54)=8.86, p<.01\right]$, while the single-stimulation response condition, SS, was least affected $\left[\mathrm{q}_{\mathrm{r}}(4,54)=2.90, \mathrm{p}<.05\right]$. This Speed-Accuracy by Response Condition interaction was significant $[F(2,54)=4.61, p<.01]$. This interaction can be attributed to the diminished speed-accuracy effect in the SS-C response condition. An analysis of only double-stimulation conditions in Table 3 failed to reveal a significant interaction $[\mathrm{F}(1,36)=2.93$, $\mathrm{p}>.05]$. As is evident in Fig. 3, an interaction was found between speed-accuracy condition and ISI $[F(4,216)=10.75, p<.01]$. This reflects the finding that percentage correct remained relatively constant under accuracy payoff until ISI dropped to $33 \mathrm{msec}$, while under speed payoff a continuous drop in accuracy was found as ISI decreased from $528 \mathrm{msec}$. No other significant two-way interactions with speed-accuracy condition were found.

As ISI decreased, an expected drop in accuracy was observed. Thus, neither response speed nor accuracy was held constant at the expense of the other as ISI changed. Comparison of $\mathrm{RT}_{1}$ and percentage correct curves for Groups DS-1[SPEED] and DS-1 [ACCUR] at each ISI level reveal a speed-accuracy tradeoff, since speed and accuracy vary inversely. A similar conclusion may be drawn from comparison of Groups DS-2[SPEED] with DS-2[ACCUR] and SS[SPEED] with SS [ACCUR]. Inspection of these sets of curves shows that the greatest range of SAT occurred at the shortest ISIs, where both $\mathrm{RT}_{1}$ and percentage correct radically shifted as speed-accuracy condition varied. However, it is at these short ISIs that information useful in selecting the correct R1 can be accumulated for the briefest interval. Thus, this finding is contrary to predictions of stimulus sampling CRT models which assume that $\mathrm{S}$ generates

Table 3

Proportion of Correct First Responses for All Combinations of Response Condition and Speed-Accuracy Condition

\begin{tabular}{lcccc}
\hline $\begin{array}{c}\text { Speed- } \\
\text { Accuracy }\end{array}$ & \multicolumn{3}{c}{ Response Condition } & \\
\cline { 2 - 4 } Condition & SS & DS-1 & DS-2 & Mean \\
\hline Accuracy & .99 & .96 & .97 & .97 \\
Speed & .94 & .85 & .78 & .86 \\
Mean & .97 & .90 & .87 & .91 \\
\hline
\end{tabular}


SATs by varying the duration over which information is accumulated.

Figure 3 reveals that an observed interaction between response condition and $\operatorname{ISI}[F(8,216)=14.17, p<.01]$ arises from the lack of ISI effect in the yoked single-stimulation groups, as would be expected. No other significant two-way interactions with ISI were found. Though $\mathrm{RT}_{1}$ decreased slightly as FP lengthened, R1 accuracy was unaffected by $F P[F(3,162)=2.05$, $\mathrm{p}>.05]$. A deadline model of CRT might predict error rates to increase with $\mathrm{FP}$, since the response deadline is met more frequently on long FP trials.

Table 3 shows that the single-stimulation response condition was more accurate than either double-stimulation response condition. Response Condition DS-2 was least accurate. Because of the significant Response Condition by Speed-Accuracy Condition interaction, differences among response conditions were tested within speed-accuracy level. Multiple $t$ tests within the accuracy condition showed no significant differences among the three response conditions. However, under speed payoff all response conditions differed from each other at the .05 level of significance or better by Newman-Keuls test $\left[\mathrm{ar}_{\mathrm{r}}(2,54) \geqslant\right.$ $\left.3.50, \mathrm{p}<.05 ; \mathrm{q}_{\mathrm{r}}(3,54)=7.39, \mathrm{p}<.01\right]$.

The three-way interaction shown in Fig. 3, Speed-Accuracy Condition by Response Condition by ISI, was significant $[F(8,216)=2.87, p<.01]$. Of particular interest in Fig. 3 is the curve for Group DS-2[SPEED]. Though $\mathrm{RT}_{1}$ was not affected by ISI in Group DS-2[SPEED], Fig. 3 shows that percentage of correct first responses was strongly affected. Indeed, this group's performance, in terms of accuracy, showed the greatest decrement as ISI was reduced. In general, groups showing small effects of ISI upon $\mathrm{RT}_{1}$ exhibited large effects of ISI upon R1 accuracy. Thus, all double-stimulation groups were adversely affected by

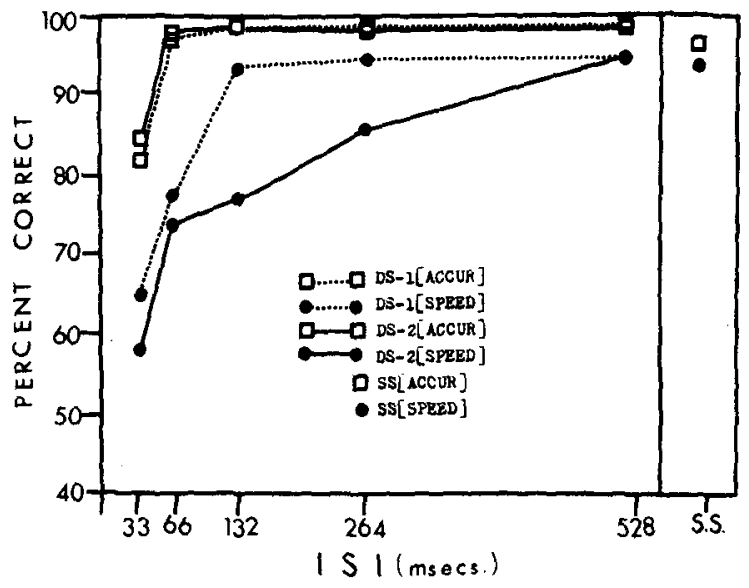

Fig. 3. First response accuracy as a function of ISI for double-stimulation groups. Since accuracy for single-stimulation groups was constant over yoked pseudo-ISI blocks, all data from each SS group have been combined into a single mean value presented in the right panel.
Table 4

Mean Rates of Information Transmission $\left(\mathrm{H}_{\mathrm{t}} / \mathrm{Sec}\right)$ as a Function of ISI for All Groups

\begin{tabular}{lrrrrr} 
& \multicolumn{5}{c}{ ISI (Msec) } \\
\cline { 2 - 6 } & 33 & 66 & 132 & 264 & 528 \\
\hline SS[ACCUR] & 2.76 & 2.71 & 2.77 & 2.75 & 2.75 \\
SS[SPEED] & 2.24 & 2.16 & 2.35 & 2.43 & 2.45 \\
DS-1[ACCUR] & .75 & 2.11 & 2.33 & 2.41 & 2.29 \\
DS-1[SPEED] & .22 & .96 & 1.94 & 2.15 & 2.33 \\
DS-2[ACCUR] & .96 & 2.25 & 2.54 & 2.44 & 2.33 \\
DS-2[SPEED] & .22 & .88 & 1.17 & 1.71 & 2.28 \\
\hline
\end{tabular}

Note-Table entries are in bits/second.

short ISIs, although the form of this performance decrement shifted between increased $\mathrm{RT}_{1}$ and decreased accuracy, primarily as a function of task speed-accuracy demands.

Transmitted Information Rate. While Ss in the present study did not produce SATs which held either speed or accuracy constant across ISI, perhaps some other performance measure was stable. One possible measure is rate of information transmission, $\mathrm{H}_{t} / \mathrm{sec}$, expressed in bits per second. Swanson and Briggs (1969) have suggested that Ss vary both speed and accuracy to maintain constant $\mathrm{H}_{\mathrm{t}} / \mathrm{sec}$. Mean $\mathrm{H}_{\mathrm{t}} /$ response was computed for each $\mathrm{S}$ at each ISI and then divided by that $\mathrm{S}$ 's mean $\mathrm{RT}_{1}$ (pooled error and correct responses) for that ISI to obtain mean $\mathrm{H}_{\mathrm{t}} / \mathrm{sec}$. This measure is shown in Table 4 for each combination of speed-accuracy condition, response condition, and ISI. Ss in different experimental groups did not produce equal $\mathrm{H}_{\mathfrak{t}} / \mathrm{sec}$ across changes in speed-accuracy condition. In terms of $\mathrm{H}_{\mathrm{t}} / \mathrm{sec}$, all double-stimulation groups showed performance decrements as ISI decreased. In addition to' the ISI effect, speed payoff produced a performance decrement relative to accuracy payoff. An analysis of variance conducted on $\mathrm{H}_{\mathfrak{t}} / \mathrm{sec}$ data revealed significant effects of speed-accuracy condition $[F(1,54)=16.28$, $\mathrm{p}<.001]$, response condition $[\mathrm{F}(2,54)=14.75$, $\mathrm{p}<.001]$, and ISI $[\mathrm{F}(4,216)=63.77, \mathrm{p}<.001]$. The effect of short ISI was more detrimental under speed payoff than under accuracy payoff $[\mathrm{F}(4,216)=7.62$, $\mathrm{p}<.001]$. Increases in response speed could not adequately compensate reductions in response accuracy, especially at short ISIs.

\section{Effects on the Second Response}

R2 Latency. Also displayed in the right panel of Fig. 2 are $\mathrm{RT}_{2}$ curves as a function of ISI for both DS-2 groups. Second response latency was strongly affected by speed-accuracy condition $[F(1,18)=12.80$, $\mathrm{p}<.001]$. Furthermore, short ISI increased $\mathrm{RT}_{2}$ more under speed payoff than under accuracy pay off $[F(4,72)$ $=7.07, \mathrm{p}<.01]$. The increase in $\mathrm{RT}_{2}$ for Group DS-2[SPEED] does not appear to reflect a PRP effect, since $\mathrm{RT}_{2}$ barely rises above the single-stimulation control RT of Group SS[SPEED]. At long ISIs $\mathrm{RT}_{2}$ for 
Group DS-2 [SPEED] became extremely fast. Perhaps Ss in this group attempted to time $\mathrm{R} 2$ to occur a fixed interval after $\mathrm{R} 1$ and did not process $\mathrm{S} 2$ information. Examination of the number of anticipatory $R 2 s$ revealed none at ISI $<264 \mathrm{msec}$ but a relatively large number (57 for Group DS-2[SPEED]) at the two longest ISIs. The number of premature R2s increased from 11 to 57 as performance emphasis shifted from accuracy to speed. This is interesting since the same high penalty for anticipations was present under both speed payoff and accuracy payoff. Also of interest is the extreme elevation of $\mathrm{RT}_{2}$ at ISI $=33 \mathrm{msec}$ for Group DS-2[ACCUR]. Since the same payoff matrix was applied to both R1 and R2 and since R2 did not require a decision once $\mathrm{R} 1$ had been selected, it might have been expected that $\mathrm{RT}_{2}$ would be no slower than $\mathrm{RT}_{1}$.

No $R 2$ accuracy data is presented, since the accuracy of R2 was completely determined by the accuracy of $\mathrm{R} 1$. If $\mathrm{S}$ made an incorrect $\mathrm{R} 1$, he could not make a correct R2 because only one response per key was allowed on each trial.

\section{DISCUSSION}

\section{Empirical Assessment}

The present experiment has shown that in double stimulation short ISIs are consistently associated with performance decrement. However, the data from Group DS-2[SPEED] demonstrate that this limitation need not necessarily show itself in the form of slowed reactions. The present results show that Ss are able to shift the form of this limitation in such a way as to minimize its impact on task performance criteria. Thus, when errors were costly, decreasing ISI primarily affected latency. However, when response speed was important, the performance decrement associated with short ISIs shifted to increased error rate.

While the present study confirms previous double-stimulation studies, which show $\mathrm{RT}_{1}$ to increase as ISI decreases (e.g., Kantowitz, 1969; Nickerson, 1967), it forces reevaluation of these results. These $\mathrm{RT}_{1}$ effects are not necessarily a consequence of information overload. Rather, they may reflect both processing limitations and the operation of strategies adopted by S. In the present study, $\mathrm{RT}_{1}$ was not affected by ISI in Group DS-2 [SPEED]; also, $\mathrm{RT}_{1}$ effects were relatively less in Group DS-1[SPEED] than in Group DS-1 [ACCUR]. Thus, it appears that $\mathrm{RT}_{1}$ delays are at least partially under S's control.

The present results strengthen the conjecture that dichotomies described by Helson and Steger (1962) and Nickerson (1967) may have resulted from between-Ss variation in SAT. Ss who were only slightly affected by ISI may have emphasized response speed rather than accuracy, thus avoiding $\mathrm{RT}_{1}$ delays at short ISIs. One would expect to also find this difference reflected in error rates according to the results of the present study. However, such data is often lacking. The present results emphasize the importance of this information in interpreting double-stimulation paradigms but found no relation between this increase and ISI. It seems likely that a relation between error rates and ISI would have appeared had this been examined.

In agreement with findings of Kantowitz (1969, 1972), the present experiment found error $R_{1}$ to be slower than correct $\mathrm{RT}_{1}$ in most double-stimulation groups. Group DS-2[SPEED] produced an exception to this general double-stimulation result, since error responses were significantly faster than correct first responses. In contrast to the double-stimulation results, single-stimulation groups produced faster error than correct responses. These single-stimulation results agree with those of Yellott (1971) and Hale (1969). These comparisons suggest that different processes are dominant in single- and double-stimulation CRT tasks, so that a single parsimonious theory of CRT performance may be incapable of dealing with both single- and double-stimulation paradigms.

Though Ss apparently traded response speed for accuracy, the form of this tradeoff did not produce constant performance in terms of latency, accuracy, or information transmission rates. However, this in part may reflect the use of unpracticed Ss in the present study. Ss' difficulty in maintaining constant $\mathrm{H}_{\mathrm{t}} / \mathrm{sec}$ appeared to result from an inability to increase speed enough to compensate losses in response accuracy, especially at short ISI. Fitts (1966) found maximum information transmission rates when response equivocation reached approximately $15 \%$ of total possible $\mathrm{H}_{\mathrm{t}}$. The present study shows that any response equivocation produces a decrease in $\mathrm{H}_{t} / \mathrm{sec}$. This is, however, consistent with results of Hale (1969). Both the present experiment and that of Hale (1969) were low stimulus and response information tasks (1 bit), while Fitts (1966) used higher levels (3.9 bits). It may be that increases in speed adequately compensate nonzero response equivocation only when high information load tasks are involved. Howell and Kreidler (1963) suggested that reduction in accuracy would be beneficial only in difficult, complex tasks. The present results substantiate that conjecture.

The first response became faster as FP increased from 1.5 to $2.5 \mathrm{sec}$. This result agrees with the single-stimulation data of Ollman and Billington (1972), who interpreted this FP effect as evidence for a deadline model of CRT. However, futher examination of the present results showed the FP effect on first response accuracy was contrary to predictions of a deadline model; accuracy was unrelated to FP.

Comparison of Response Conditions DS-1 and DS-2 under both speed and accuracy conditions showed DS-2 to be consistently faster. Thus, Herman and McCauley's (1969) result is confirmed: $\mathrm{RT}_{1}$ is faster if two responses rather than just one are required. This finding cannot be attributed to SAT differences as originally conjectured. Quite possibly the apparent high level of 
response grouping in Response Condition DS-2 may play a role in obtaining this result.

While the focus of the present study was on $\mathrm{R} 1$ effects, results also showed $\mathrm{R} 2$ performance to be strongly affected by SAT. The changes in S2-R2 processing attributable to SAT cannot easily be compared to changes in S1-R1 processing since the uncertainty associated with these two components differed. Although $\mathrm{RT}_{2}$ curves of similar shape were obtained for Groups DS-2[SPEED] and DS-2[ACCUR], these curves may reflect different processes. The DS-2[ACCUR] curve appears to represent a typical PRP effect, since at short ISIs $\mathrm{RT}_{2}$ is elevated far above a single-stimulation control RT. Indeed, at short ISIs R2 which did not require a decision once $R 1$ had been selected was slower than $R 1$ which did require a 1-bit decision. Furthermore, since the same payoff matrix scored both R1 and R2, this R2 delay was just as costly to $S$ as $R 1$ delay.

For Group DS-2[SPEED], however, $\mathrm{RT}_{2}$ barely exceeded the single-stimulation control RT, SS [SPEED], even at the shortest ISIs. Again, however, differences in stimulus and response uncertainty between R1 of the control and R2 do not allow the conclusion that $\mathrm{S} 2-\mathrm{R} 2$ processing was unaffected by $\mathrm{S} 1$ or R1. It is likely that comparison of $\mathrm{RT}_{2}$ at short ISIs with an event-certain single-stimulation $\mathrm{RT}$ would have yielded evidence of $\mathrm{RT}_{2}$ elevation. The $\mathrm{RT}_{2}$ data for Group DS-2[SPEED] suggest that Ss in this group were not fully processing $S 2$, but rather were executing a "grouped" response as soon as S1 arrived. Ss apparently attempted to time their responses to coincide with S2 onset. The first stimulus may have served as a temporal marker to initiate the timed interval, especially since blocked rather than random ISI presentation was used.

\section{Theoretical Assessment}

The present experiment has shown that the effects of SAT and double-stimulation variables such as ISI and response condition are not additive. This makes the simple application of single-stimulation SAT models to each component decision in the double-stimulation paradigm less appealing. While such an approach might lead one to predict overall elevation or decrease of RT curves as a function of SAT, results such as the elimination of ISI effects under speed conditions would be unexplained. In addition to this general difficulty in applying single-stimulation SAT models to double stimulation, specific portions of the present data are difficult to reconcile with stimulus sampling, fast guess, or response conflict type models.

Stimulus Sampling Models. The present study showed that changes in speed-accuracy condition produced the greatest performance changes at short ISIs, and this suggests that Ss were varying SAT over the widest range at these ISIs. Such a finding appears contrary to stimulus sampling models which predict greatest range of SAT at longest ISIs, since at longer ISIs Ss have the option of accumulating information for longer durations. Such a model might explain present findings by requiring that Ss continue to sample display information for a period of time after S2 onset. However, information sampled after S1 and S2 have appeared cannot aid in determining the correct R1. Thus, time is being expended with no gain in accuracy-a highly inefficient strategy.

Fast Guess Models. Fast guess models find difficulty with three aspects of the double-stimulation error RT data. First, the present experiment found error RT to exceed correct RT in double stimulation. Most fast guess models predict faster errors since these responses are generally based upon very minimal information processing. A recent variant of the fast guess models, the deadline model of Ollman and Billington (1972), is still unable to account for this double-stimulation result. According to this model, $\mathrm{S}$ attempts to set a response deadline which elapses shortly after the longest FP. The setting of this deadline depends on the speed-accuracy demands of the task. If the deadline expires before stimulus processing is complete, $\mathrm{S}$ simply executes a fast, often inaccurate guess response. However, this should occur most frequently at the longest FP. Thus, on long FP trials short latency responses are likely to be errors that result from deadline expiration rather than completion of stimulus processing. On short FP trials the opposite is true; generally stimulus processing will be complete before the deadline is reached. Thus, short latency responses will more likely be correct than will slow responses. The exact relation among accuracy, latency, and FP will depend upon the variability of the deadline setting and stimulus processing time. The present experiment showed that double-stimulation error responses were generally slower than correct responses at all foreperiods. Indeed, the difference between correct and error response latencies seemed unaffected by FP.

Second, the present double-stimulation data show errors to be a highly variable response type with a fairly uniform latency density function (except for the peaked function found for Group DS-2[SPEED]). Even though Group DS-2 [SPEED] did generate a unimodal function, results revealed the variance of error $\mathrm{RT}_{1}$ to exceed the variance of correct $\mathrm{RT}_{1}$. This finding also seems contrary to most fast guess SAT models, since such models predict error RT variance to be less than correct RT variance. This follows from the assumption that errors represent a single response type (guesses), while correct RTs result from both slow stimulus-controlled responses and fast guesses. To some degree, the additional error variance found in the present study may be attributed to the smaller sample sizes from the error distributions. However, confidence interval estimates, which took this factor into account, still showed error $\mathrm{RT}_{1}$ variance to considerably exceed correct $\mathrm{RT}_{1}$ variance. A deadline model might be able, however, to account for this error variance by assuming that the deadline setting is noisy. Since error RT is related to this 
deadline, the variance of the error latency density functions might be inflated by the variance of the deadline setting.

Third and finally, the finding that changes in error $\mathrm{RT}_{1}$ exceed changes in correct $\mathrm{RT}_{1}$ as payoff ${ }^{*}$ condition varies is difficult to reconcile with most fast guess models. Such models predict error $\mathrm{RT}_{1}$ shifts to be less than correct $\mathrm{RT}_{1}$ shifts, since error RT primarily reflects a uniform response type-guesses. This difficulty cannot be overcome by a "slow guess" model in which latency of guesses exceeds that of SCRs. Fast guess models might attempt to deal with this finding by assuming that Ss change preprogrammed guess response latency on the basis of speed-accuracy stress, so that guesses take longer under accuracy payoff. However, such a model is closer to a stimulus sampling model, since SAT is accounted for by distribution changes rather than by guessing probability. The generalized fast guess model proposed by Yellott (1971), which allows variation in both the latency of correct and incorrect stimulus-controlled responses and the accuracy of SCRs, also meets this same obstacle. Attempts to explain present findings by allowing greater latency for incorrect SCRs relative to correct SCRs are insufficient, since this would predict a bimodal error RT distribution with fast errors arising from guesses and slow errors from incorrect SCRs. Only unimodal distributions for correct and incorrect $\mathrm{RT}_{1}$ were obtained, even when the distribution was limited to the shortest ISI at which the greatest number of errors occurred. Thus, variation in probability of correct and incorrect SCRs must also be postulated. However, such a manipulation puts the burden of SAT on correct and incorrect SCRs rather than on the relationship between guesses and SCRs; therefore, such a model is best considered a stimulus sampling model with occasional guesses rather than as a fast guess model. Indeed, Yellott (1971) never gives much consideration to this completely generalized "fast guess" model.

This finding of greater shift of error $\mathrm{RT}_{1}$ than correct $\mathrm{RT}_{1}$ is also contrary to a deadline CRT model. Such a model assumes that $\mathrm{S}$ attempts to set his deadline such that it expires just after the longest FP but before (longest FP + time required for stimulus processing). Thus, error RT should be roughly bounded and should exhibit less variation than correct RT.

Response Conflict Models. Some results of the present experiment are consistent with an extended version of response conflict theory (Herman \& Kantowitz, 1970; Kantowitz, 1974; Reynolds, 1966). According to this extended model, conflict during response selection always yields a performance decrement, but this may be exhibited as either decreased accuracy or increased latency, depending upon S's SAT strategy. Such a model is consistent with the finding that the widest range of SAT occurred when conflict was presumably greatest-at short ISIs (Herman \& Kantowitz, 1970; Reynolds, 1966). Furthermore, this model assumes that, overall, response selection is more difficult and slower under high conflict. Error responses which, a fortiori, result from high conflict trials should, therefore, be slower than correct responses at any fixed level of SAT adopted by $\mathrm{S}$. This, however, presents a problem. As performance emphasis shifts from accuracy to speed, the data show that Ss generate more slow errors. Examination of the present results resolves this paradox; the latency of error responses drops sharply as speed emphasis increases. This results in an overall decrease in average response speed when correct and error responses are combined.

Herman and McCauley (1969) found $\mathrm{RT}_{1}$ to be faster in a DS-2 than DS-1 response condition. They interpreted this finding, replicated in the present experiment, in terms of greater conflict during response selection in the DS- 1 than DS-2 response condition. The basis for this interpretation was that excitatory and inhibitory response tendencies were juxtaposed in DS-1 (since $S$ made an overt response to $S 1$ but had to inhibit a response to S2), while in DS-2 two excitatory response tendencies were juxtaposed (since overt responses were required to both $\mathrm{S} 1$ and S2). The former situation was thought to produce more conflict during response selection than the latter. If, indeed, more conflict were present during response selection in the DS-1 than DS-2 response condition, the response conflict SAT model would predict an interaction between speed-accuracy condition and response condition: Changes in speed-accuracy condition should produce greater effects in Response Condition DS-1 than DS-2. No evidence for such an interaction was found when double-stimulation latencies (Table 1) or error rates (Table 2) were examined, and this presents a difficulty for response conflict SAT models.

Because of differences between single- and double-stimulation error latency results, the present experiment suggests that single- and double-stimulation performance may reflect different processes. In single stimulation the data presented here seem generally consonant with both stimulus sampling and fast guess SAT models. However, these models, as well as the extended response conflict model, are unable to deal with all the double-stimulation SAT effects. Perhaps a model encompassing more than one SAT mechanism is required. Yellott (1971), for instance, has proposed a generalized fast guess model in which stimulus-controlled response latency might theoretically provide a secondary locus of SAT effects in addition to that provided by varying the proportion of guess and stimulus-controlled responses. Finally, difficulty with the extended response conflict model is predicated on an assumption that changes in response condition altered conflict level during response selection. If this assumption is incorrect, the response conflict model might yet prove a viable double-stimulation SAT model.

\section{REFERENCES}

Annett, J. Payoff: A neglected factor in reaction time measurement. Quarterly Journal of Experimental Psychology, $1966,18,273-274$. 
Edwards, W. Costs and payoffs are instructions. Psychological Review, 1961, 68, 275-284.

Edwards, W. Optimal strategies for seeking information: Models for statistics, choice reaction times, and human information processing. Joumal of Mathematical Psychology, 1965, 2, 312-329.

Fitts, P. M. Cognitive aspects of information processing: III. Set for speed versus accuracy, Journal of Experimental Psychology, 1966, 71, 849-857.

Hale, D. J. Speed-error tradeoff in a three-choice serial reaction task. Journal of Experimental Psychology, 1969, 81, 428-435.

Helson, H., \& Steger, J. A. On the inhibitory effects of a second stimulus following the primary stimulus to react. Journal of Experimental Psychology, 1962, 64, 201-205.

Herman. L. M. Effects of second signals on response time to first signals under certainty and uncertainty. Journal of Experimental Psychology, 1969, 80, 223-229.

Herman, L. M., \& Kantowitz, B. H. The psychological refractory period effect: Only half the double-stimulation story? Psychological Bulletin, 1970, 73, 74-88.

Herman, L. M. \& McCauley, M. E. Delay in responding to the first stimulus in the "psychological refractory period" experiment: Comparisons with delay produced by a second stimulus not requiring a response. Journal of Experimental Psychology, 1969, 81, 344-350.

Howell, W. C., \& Kreidler, D. L. Information processing under contradictory instructional sets. Journal of Experimental Psychology, 1963, 65, 39-46.

Kantowitz, B. H. Double-stimulation with varying response information. Journal of Experimental Psychology, 1969, 82, 347-352.

Kantowitz, B. H, Response conflict mechanisms in double stimulation. Presented at information processing/double stimulation symposium, American Psychological Association, September 1972.

Kantowitz, B. H. Double stimulation. In B. H. Kantowitz (Ed.) Human information processing: Tutorials in performance and cognition. Potomac, Md: Erlbaum, 1974.

Knight, J. L. The speed-accuracy tradeoff in double stimulation: Effects on the first response. Unpublished Master's thesis, Purdue University, January 1972.

Nickerson, R. S. Psychological refractory phase and the functional significance of signals. Journal of Experimental
Psychology, 1967, 73, 303-312

Ollman, R. T. Fast guesses in choice reaction time. Psychonomic Science, $1966,6,155-156$

Ollman, R. T., \& Billington, M. J. The deadline model for simple reaction times. Cognitive Psychology, 1972, 3, 311-336.

Pachella, R. G., \& Pew, R. W. Speed-accuracy trade-off in reaction time: Effect of discrete criterion times. Journal of Experimental Psychology, 1968, 76, 19-24.

Reynolds, D. Time and event uncertainty in unisensory reaction time. Journal of Experimental Psychology, 1966, 71, 286-293.

Stone, M. Models for choice reaction time. Psychometrika, 1960 , $25,251-260$.

Swanson, J. M., \& Briggs, G. E. Information processing as a function of speed versus accuracy. $J$ ournal of Experimenta Psychology, 1969, 81, 223-229.

Yellott, J. I., Jr. Correction for guessing and the speed-accuracy trade-off in choice reaction time. Journal of Mathematical Psychology, 1971, 8, 159-199.

\section{NOTE}

1. At long ISIs performance may be both faster and more accurate than at short ISIs because response conflict, a primary source of task difficulty, is reduced. At short ISIs, where response conflict and, consequently, task difficulty are great, the necessity for $S$ to trade-off one performance dimension against the other would increase.

2. Corresponding tables and figures based only on correct responses (Knight, 1972) were nearly identical and, therefore, not included here. In addition, tables and figures based only on correct responses could have included unknown proportions of fast correct guesses and, therefore, be somewhat misleading. Where possible, analyses were performed both on pooled correct and error data and on correct responses only. Unless otherwise noted, the results of the analy ses were similar.

(Received for publication November 26, 1973; accepted January $1,1974$. .) 